\title{
Incompressible-compressible transition in falling granular jets
}

\author{
G. Prado, Y. Amarouchene ${ }^{(a)}$ and H. Kellay \\ Université Bordeaux 1, Laboratoire Ondes et Matière d'Aquitaine, CNRS UMR 5798 \\ 351 cours de la libération, 33405 Talence, France, EU
}

PACS 45.70.-n-Granular systems

\begin{abstract}
Through a systematic experimental investigation of the behavior of falling granular jets under the action of gravity for different particle sizes, funnel diameters and ambient air pressures, necessary conditions to obtain incompressible granular jets are identified. A transition from compressible (characterized by a significant density decrease along the propagation) to incompressible granular jets (characterized by a constant density) is observed. This transition depends solely on the aspect ratio between the diameter of the particles and the diameter of the funnel. Surprisingly, the incompressible liquid-like behavior observed here seems to find its origin in the balance between the heat flux and the dissipation in the funnel independently of the ambient fluid pressure. A simple granular hydrodynamic model provides a good description of the transition.
\end{abstract}

Particles or grains flowing from hoppers and silos have attracted the attention of scientists since the seminal work of Hagen [1-3]. Contrary to molecular fluids, the flow rate is constant and does not depend on the height of the granular layer in sharp contrast with the Torricelli law describing fluids emptying from vessels. It is also known that the mass flow rate is mainly fixed by the ratio between the aperture at the exit $D$ and the particles diameter $d$. Beverloo proposed an empirical expression $(Q \sim(D-$ $k d)^{5 / 2}$ with $k \sim 3-5$ with a characteristic velocity scaling as $\sqrt{g D} ; g$ being the gravity acceleration) that is widely used for large aspect ratios $D / d$. However, deviations are observed for smaller aspect ratios [4-6]. It was anticipated that this deviation may be due to a lower density near the exit $[7,8]$.

Recently, several studies focused on the properties of granular jets falling under the action gravity from hoppers or funnels, the main interest lying in describing the surface properties of these jets $[9,10]$. An analog of the surface tension has been characterized but the bulk properties remain poorly studied [11-16]. It is the purpose of the present paper to investigate the bulk properties of granular jets falling under gravity. These jets were observed to thin as they propagate downstream, just like liquid jets, with a roughly constant density suggesting incompressible-like behavior [14-16]. However, less attention has been paid to the understanding of the conditions that set the incompressible nature of granular jets. Through a systematic

\footnotetext{
(a) E-mail: y . amarouchene@loma.u-bordeaux1.fr
}

study of the effect of the particle size, ambient pressure, funnel diameter on the thinning properties of granular jets, a clear "Incompressible-Compressible" transition is observed in our experiments. The control parameter for this transition turns out to be the aspect ratio $D / d$. This transition occurs for low aspect ratios near 15 for which the departure from the Beverloo law occurs. The transition observed here can be rationalized using a continuum hydrodynamic model for granular media by taking into account the role of the boundaries.

In these experiments, we have used various funnels and granular materials. The funnels are all open topped and made of plastic or glass with an exit half-angle of the order of 2.5 degrees. In the following, the exit diameter of the funnel is noted $D$ (varying from a few $\mathrm{mm}$ to $35 \mathrm{~mm}$ ). The granular materials used are made of glass spheres $\left(\rho_{B}=2500 \mathrm{~kg} \cdot \mathrm{m}^{-3}\right)$ of mean diameter ranging from $d \approx 79.5 \mu \mathrm{m}$ to $d \approx 1 \mathrm{~mm}$ (standard deviation $\delta d \sim 25 \mu \mathrm{m})$. Movies of the flowing granular material are made with a Phantom v640 camera with a typical acquisition rate of 4000 frames $\cdot \mathrm{s}^{-1}$ and a typical spatial resolution of $20 \mu \mathrm{m} \cdot$ pixel $^{-1}$. Experiments conducted under vacuum are done using a $2 \mathrm{~m}$ long and $9 \mathrm{~cm}$ wide transparent PMMA cylinder sealed at its top and its bottom. Measurements of the jet diameter and the beads velocities are carried out using various image processing routines including PIV and PTV (Particle Imaging Velocimetry and Particle Tracking Velocimetry, respectively). Experimental details can be found in previously published papers $[15,16]$. 


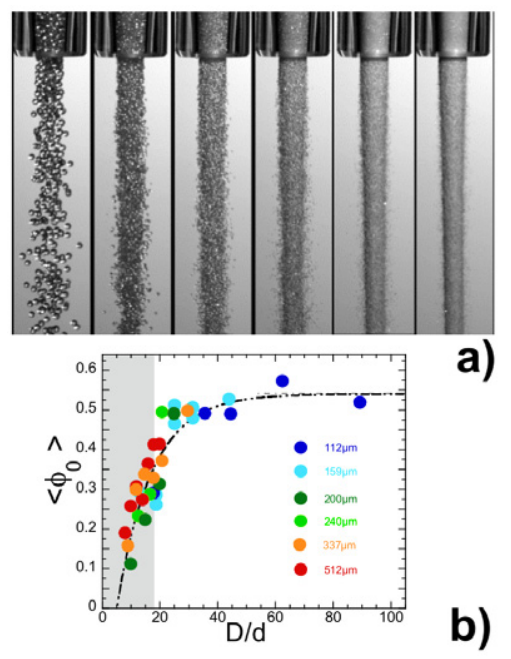

Fig. 1: (Colour on-line) (a) Snapshots of granular jets near the exit of a funnel (diameter $D=5 \mathrm{~mm}$ ) for different grain sizes $d$ (from left to right $1000 \mu \mathrm{m} ; 512 \mu \mathrm{m} ; 337 \mu \mathrm{m} ; 240 \mu \mathrm{m} ; 159 \mu \mathrm{m}$ and $112 \mu \mathrm{m})$. Note that the granular jet thins for small grains, $d<337 \mu \mathrm{m}$. For larger grains, $d \sim 337 \mu \mathrm{m}$, the thinning does not occur. For even larger grains, $d=1000 \mu \mathrm{m}$, the jet spreads out and becomes less dense. (b) Mean volume fraction $\left\langle\phi_{0}\right\rangle$ estimated by the simultaneous measurement of the mass flux $Q$ and the mean velocity $\left\langle V_{0}\right\rangle$ at the exit of the funnel for different grain sizes $d v s$. the aspect ratio $D / d$. Note the decrease of $\left\langle\phi_{0}\right\rangle$ for $D / d$ below 15 while for higher aspect ratios $(D / d>20)$, a saturation at $\left\langle\phi_{0}\right\rangle \sim 0.5$ is observed.

Figure 1(a) shows snapshots of granular jets falling under the action of gravity for different grain sizes $d$ while the funnel diameter $D$ is kept constant $(D=5.3 \mathrm{~mm})$. Note that the granular jet thins for small grains $(d<$ $337 \mu \mathrm{m}$ or $D / d>15)$. However for larger grains the thinning does not occur $(d>512 \mu \mathrm{m}$ or $D / d<10)$, and for even larger grains the jet spreads out and becomes less dense. If we keep the grain diameter $d$ fixed, a similar behavior is observed while varying the funnel diameter $D$ with thinning jets for large $D$ and jets that spread out for small $D$. The jets for $D / d<10$ are clearly less dense than for $D / d>10$. Furthermore, they seem to become less dense as they propagate. The mean density or volume fraction $\left\langle\phi_{0}\right\rangle$ at the exit can be estimated by the simultaneous measurement of the mass flux $Q$ and the mean velocity $\left\langle V_{0}\right\rangle$ (which is close to the velocity of grains measured near the wall inside the funnels and is of the order of $10 \mathrm{~cm} / \mathrm{s})$ at that location. Using mass conservation, we have $\left\langle\phi_{0}\right\rangle=4 Q /\left(\rho_{B}\left\langle V_{0}\right\rangle \pi D^{2}\right)$. The results obtained for $\left\langle\phi_{0}\right\rangle$ are shown in fig. 1(b) for different aspect ratios $(D / d)$ : for fixed $D$ and variable $d$ or fixed $D$ and variable $d$. Our main observation is the decrease of the mean volume fraction below $D / d \sim 15$. For higher aspect ratios $(D / d>20)$, a saturation at $\left\langle\phi_{0}\right\rangle \sim 0.5$ is reached. This is close to the value expected for random loose packings.

An analysis of the granular jets diameter $v s$. the distance from the exit $z$ provides a means to characterize the

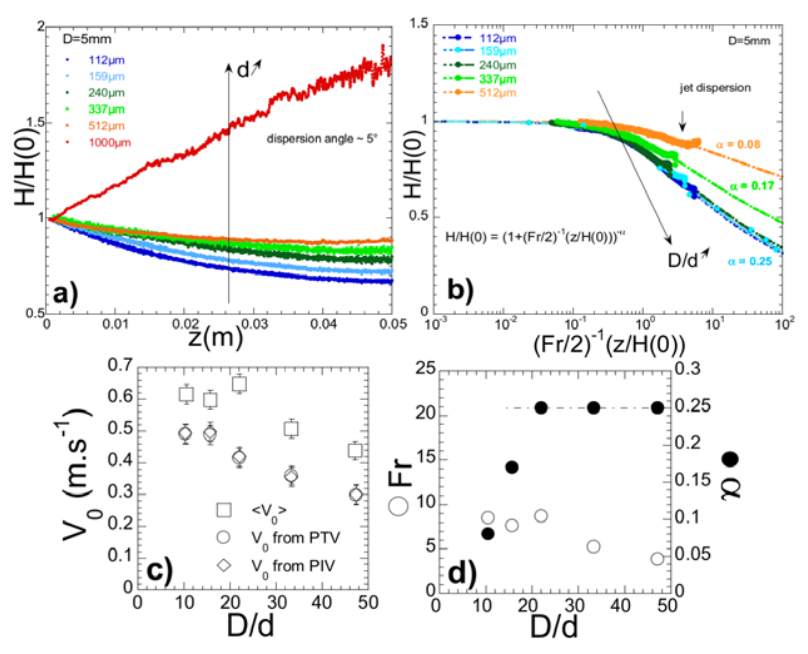

Fig. 2: (Colour on-line) (a) Granular jet diameter vs. the axial distance $z$ for $D=5 \mathrm{~mm}$ and various grain sizes $d$. (b) Granular jet diameter vs. the rescaled axial distance $(F r / 2)^{-1} z / H(0)$ for $D=5 \mathrm{~mm}$ and various grain sizes $d$. (c) $V_{0}$ extracted from the thinning jet diameter $\left(\left\langle V_{0}\right\rangle\right)$ and from PIV and PTV measurements at the wall vs. aspect ratio $D / d$ for $D=5 \mathrm{~mm}$ and various grain sizes $d$. (d) Froude number $F r$ and compressibility index $\alpha$ vs. aspect ratio $D / d$ for the data in (b).

transition (corresponding to pictures shown in fig. 1(a). The diameters $H(z)$ are extracted using image analysis of the light transmitted through the jet. The radial intensity profile for each $z$ is adjusted by a Gibbs law or a Hyperbolic tangent profile $[15,16]$. This analysis permits to measure the thickness or diameter of the jets $H(z) v s$. distance $z$ as displayed in fig. 2(a) for a funnel diameter $D=5 \mathrm{~mm}$. Note that the thinning is less pronounced as $d$ increases. For the largest beads $(D / d=5 ; d=1 \mathrm{~mm})$, we observe expanding jets. These jets expand linearly vs. the distance $z$ with a mean angle of the order of $5^{\circ}$. For an intermediate range of aspect ratios $10<D / d<20$, the jets thin at first, but beyond a characteristic distance $z_{d}$ the jet spreads out. $z_{d}$ decreases as the aspect ratio $D / d$ decreases. For $D / d<15, z_{d}$ is close to zero.

When a liquid (density $\rho$ and surface tension $\sigma$ ) emerges from the exit of a funnel or from the opening at the bottom of a reservoir, it is accelerated downward by gravity: the velocity grows with $z$ as $V(z)=\sqrt{2 g z+\left\langle V_{0}\right\rangle^{2}}$. While the jet is stretched by gravity, it simultaneously thins. In the incompressible limit, mass conservation $\left(V(z) H^{2}(z) \sim\right.$ constant) implies that the average diameter $H$ of the jet decays with $z$ as $H(z)=H(0)\left(1+\frac{2}{F r} \frac{z}{H(0)}+\frac{1}{W e}\left(1-\frac{H(0)}{H}\right)\right)^{-1 / 4} \quad$ with $F r=\frac{\left\langle V_{0}\right\rangle^{2}}{g H(0)}$ and $W e=\frac{\rho\left\langle V_{0}\right\rangle^{2} H(0)}{2 \sigma}$ being, respectively, the Froude number (ratio inertia/gravity) and the Weber number (ratio inertia/capillarity) [17]. In the limit $W e \gg 1$ the dimater of the jet is well approximated by: $H(z)=H(0)\left(1+\frac{2}{F r} \frac{z}{H(0)}\right)^{-1 / 4}$ and is independent of both $\sigma$ and $\rho$. For instance, this condition is fulfilled for water 
jets [17]. Therefore, it is difficult to measure a surface tension from the thinning of jets in the limit $W e \gg 1$ even for a relatively high surface tension liquid like water $(\sigma=72 \mathrm{mN} / \mathrm{m})$

The granular jet diameters $H(z)$ for $D / d>15$ are well approximated by this law using an exponent of $-1 / 4$ and $\mathrm{Fr}$ as a free parameter. This indicates that the granular jet thinning is mainly affected by inertia and that capillary forces are weak as has been found before $[15,16]$ but most importantly this highlights that the packing density of the jet remains roughly constant during the free fall. As a consequence, for aspect ratios $D / d>15$, once rescaled by $\mathrm{Fr}$ and $H(0)$, all the mean profiles of the jets collapse on a master curve as shown in fig. 2(b). Here, the Froude number $\mathrm{Fr}$ contains only the mean velocity as a free parameter. The values for $V_{0}$ extracted from the Froude fitting of the normalized jet evolution along with the values measured at the surface of jet using either PIV or PTV are shown in fig. 2(c). The measurements using PIV or PTV are similar and follow the same trend as the values obtained from Froude fitting albeit smaller by a factor $2 / 3$. This suggest the existence of a non flat velocity profile. Note that for all the data presented, $H(0)$ is simply related to $D$ by the linear relation $H(0)=D-1.5 d$ while for liquids $H(0)=D$. This highlights the discrete nature of granular fluids.

For aspect ratios $D / d<15$, the thinning is less pronounced and the incompressibility condition previously described no longer holds. An alternative to describe them is to introduce a functional form $H(z)=H(0)\left(1+\frac{2}{F r} \frac{z}{H(0)}\right)^{-\alpha} \quad$ with a compressibility index $\alpha$. The volume fraction evolution is then simply given by $\phi(z)=\phi_{0}\left(1+\frac{2}{F r} \frac{z}{H(0)}\right)^{2 \alpha-\frac{1}{2}}$. For $\alpha=1 / 4$, we recover the incompressible liquid-like limit $(\phi=$ const), while for $\alpha<1 / 4$ the jets become less dense as they propagate downstream. The values of $F r$ and $\alpha$ for all the jets corresponding to fig. 2(a) are presented in fig. 2(d). Indeed, the value of $\alpha$ is constant and close to $1 / 4$ for $D / d>15$ and monotonically decreases towards zero as the aspect ratio $D / d$ decreases. The Froude number decrease as $D / d$ increases in the incompressible regime. This indicates that the average exit velocity decreases with $D / d$ for $D / d \geqslant 15$ as shown in fig. 2(c). The mean velocity displays the same trend as the velocity measured at the surface using either PIV or PTV suggesting the existence of velocity profile inside the funnel. This is to be contrasted with the results obtained for granular flows through apertures in silos where the exit velocity is of the order of $(g D)^{1 / 2}$ for a fixed grain size (see [8]).

The compressibility index $\alpha$ is shown in fig. 3 for various experimental conditions (various $D$ and $d$ ). This exponent depends only on the aspect ratio $D / d$. The surrounding medium (air) seems to have no influence on this diagram since the same results are observed under vacuum (13 mbar) both in the compressible and incompressible granular jet regimes. For $D / d>15$, other measurements made at a much lower pressure $(0.25 \mathrm{mbar})$

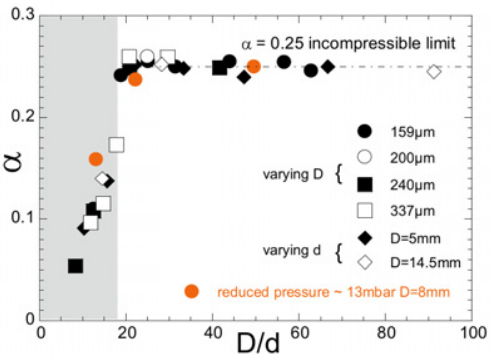

Fig. 3: (Colour on-line) Compressibility index $\alpha$ vs. the aspect ratio $D / d$ for various experimental conditions.

farther downstream corroborate this observation for the incompressible limit. Indeed the jets continue to thin as in the presence of air even at 0.25 mbar. This may suggest either a weak influence of the surface tension previously reported $[15,16]$ in the limit $W e \gg 1$ and $D / d>15$ or that the incompressible nature of a granular jet is set by other mechanisms. For instance, in a recent numerical work, it was suggested that the collimating properties of granular jets arise from dissipative inelastic collisions [18].

To gain insight into how and why the transition at $D / d \sim 15$ occurs, let us briefly summarize our reasoning to rationalize the experimental results presented here. It is known that grains close to solid boundaries in granular flows are most of the time hotter in terms of granular temperature (see GDR MiDi experiments in [4]). Furthermore, this temperature drops away from the boundaries. Thus, it is natural to expect a heat flux directed from the walls where the particles are hotter to the center of the funnel where the temperature is smaller. The grains are colder at the center of the channel because this heat flux must be balanced by dissipation. The dissipation is due to inelasticity of the collisions either between the grains or between the grains and the walls. The dissipation may also have a frictional origin. One may, according to this scenario, anticipate a denser and colder region at the center of the funnel and a less dense and hot region near the walls. If the thickness of this region near the boundary becomes of the order of the funnel diameter, one may then expect less dense jets at the exit. In order to quantify this scenario, we model the granular medium inside the funnel using a continuum approach without mean flow for simplicity. We will assume that the behavior of the jet right after the exit is dictated by the properties of the granular medium inside the funnel. Figure 4 displays a schematic of the system of interest.

Continuum modeling of granular media relies on modified versions of the Navier-Stokes equations derived from the kinetic theory of colliding inelastic hard spheres. One of the simplest sets of equations that still capture the effect of the inelastic collisions has been introduced a few years ago by Grossman, Zhou and Ben-Naim to describe the steady-state properties of granular gases in two dimensions for a wide range of densities below the close-packing density $\rho_{c}=\frac{2}{\sqrt{3} d^{2}}$ [19]. Here, in the steady 


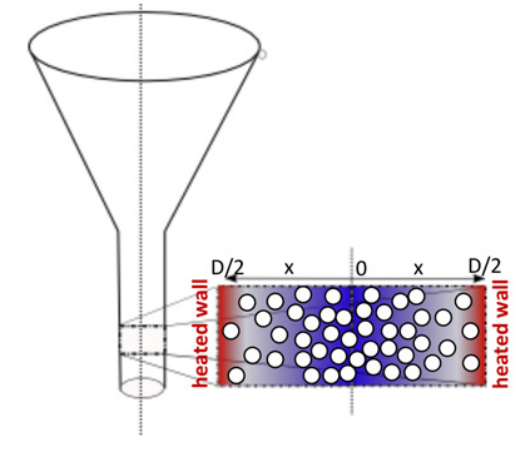

Fig. 4: (Colour on-line) Schematic of the system of interest for the numerical simulation. The length of the system is $R=D / 2$. The heated wall is at $x=D / 2$.

state, all quantities vary only in the transverse direction $x$. An energy balance relating the heat flux (from the walls towards the center) to the energy lost $I$ due to the inelasticity of collisions (denoted by the inelastic coefficient $e$ ) is given by $\frac{\mathrm{d}}{\mathrm{d} x}\left(\kappa \frac{\mathrm{d} T}{\mathrm{~d} x}\right)=I ; \kappa$ being the thermal diffusivity given by $\kappa \propto \frac{\rho(A l+d)^{2} \sqrt{T}}{l}$. Here $A=1.15$ is a dimensionless factor and $l \approx \frac{1}{\sqrt{8} \rho d} \frac{\rho_{c}-\rho}{\rho_{c}-a \rho}$ (with $a=1-$ $\sqrt{3} / 8)$ is the mean free path. The energy balance equation finally reads $\frac{\mathrm{d}}{\mathrm{d} x}\left(\frac{(\alpha l+d)^{2} \sqrt{T}}{l} \rho \frac{\mathrm{d} T}{\mathrm{~d} x}\right)=\left(1-e^{2}\right) \rho T^{3 / 2} / \gamma l$ (with $\gamma=2.26$ being the ratio of the prefactors of the thermal diffusivity and the elastic loss). These equations must be complemented by an appropriate constitutive equation relating pressure $P$, density $\rho$ and temperature $T$. For convenience, we choose the one proposed by Grossman et al. [19]: $P=\rho T \frac{\rho_{c}+\rho}{\rho_{c}-\rho}$ (a more accurate global equation of state valid close to and beyond $\rho_{c}$ has been introduced in the literature [20]). Following Janssen, the pressure $P$ is kept constant and given by $P=\frac{\langle\rho\rangle g R}{2 \mu K}$ (with Janssen coefficient $K$ and friction coefficient $\mu$ ) [4]. The order of magnitude of the dynamic Janssen pressure used for our simulation $(\mu K=1)$ is $2-3$ times higher than the value usually assumed for the static case as reported in [21]. Two boundary conditions are needed to solve numerically the problem at hand. We choose to impose a zerotemperature gradient $\frac{\mathrm{d} T}{\mathrm{~d} x}{ }_{x=0}=0$ at the center of the funnel and a fixed temperature $T$ at the boundary equal to the value we have measured experimentally as we will detail below.

The temperature near the inner wall of the funnel above the outlet is achieved by measuring the velocity of the grains using transparent glass funnels. Figure 5(a) and fig. 5(b) show the probability distribution functions of the transverse and longitudinal velocity at the wall rescaled by the standard deviation for different grain sizes and a funnel diameter $D=5.3 \mathrm{~mm}$. All the data collapse for both components of the surface velocity. An almost Gaussian behavior for the most probable events and algebraic for large velocities is observed in agreement with previous reported results [22]. The standard deviation for both components of the surface velocity $\left\langle\delta U^{2}\right\rangle^{1 / 2}$ and

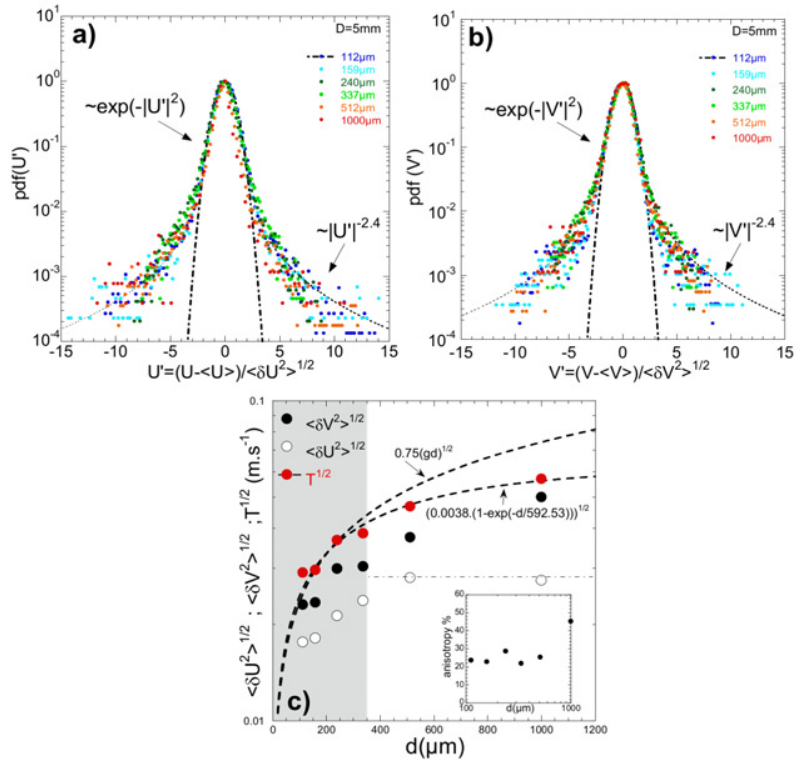

Fig. 5: (Colour on-line) Probability distribution functions of the velocity of the grains near the wall for various grain sizes and a funnel diameter $D=5 \mathrm{~mm}$ at a distance $z=-20 \mathrm{~mm}$ above the outlet. (a) Rescaled transverse component $U^{\prime}$. (b) Rescaled longitudinal component $V^{\prime}$. The thick dotted line corresponds to a Gaussian fit while the thin dotted line corresponds to a power law. (c) Standard deviation of $U, V$ and temperature $T$ vs. $d$ for $D=5 \mathrm{~mm}$; inset: anisotropy of the velocity fluctuations $v s$. $d$ under the same conditions.

$\left\langle\delta V^{2}\right\rangle^{1 / 2}$ used to collapse all the probability distribution functions shown in figs. 5(a) and (b) are displayed in fig. 5(c). The granular temperature $T$ defined using $T^{1 / 2} \equiv\left(\left\langle\delta U^{2}\right\rangle+\left\langle\delta V^{2}\right\rangle\right)^{1 / 2}$ is also displayed in fig. 5(c). The order of magnitude of $T^{1 / 2}$ is a few $\mathrm{cm} / \mathrm{s}$ and increases monotonically $v s$. the grain size $d$. The increase is close to the $(g d)^{1 / 2}$ scaling expected from dimensional arguments for the standard deviation of the velocity. A deviation is however observed for large $d(D / d<15)$. The measured temperature at the wall (necessary to impose boundary conditions in the numerical simulation) is well approximated for all grain diameters by $T=T_{\infty} \cdot\left(1-\exp \left(d / d_{c}\right)\right)$ with $T_{\infty}=0.0038$ and $d_{c}=0.592 \mathrm{~mm}$ as fitting parameters. For all the data presented here, an anisotropy of the order of $20 \%$ (defined as $\left(\frac{\left\langle\delta V^{2}\right\rangle^{1 / 2}-\left\langle\delta U^{2}\right\rangle^{1 / 2}}{\left\langle\delta V^{2}\right\rangle^{1 / 2}}\right)$ is present independently of the bead size $d$ as shown in the inset of fig. $5(\mathrm{c})$.

These measurements allow us to fix the value of the temperature at the wall. The numerical resolution using this fixed temperature $T$ measured at the wall $v s$. the grain diameter $d$ is shown in fig. 6. The density profiles vs. $x$, rescaled by the close-packing volume fraction, are shown in fig. 6(a). For all the results presented, the volume fraction at the wall is always smaller than the value at the center of the domain of calculation. A clear decrease of the volume fraction at the center of the funnel is observed for the lowest aspect ratios $D / d$, while high densities are observed 

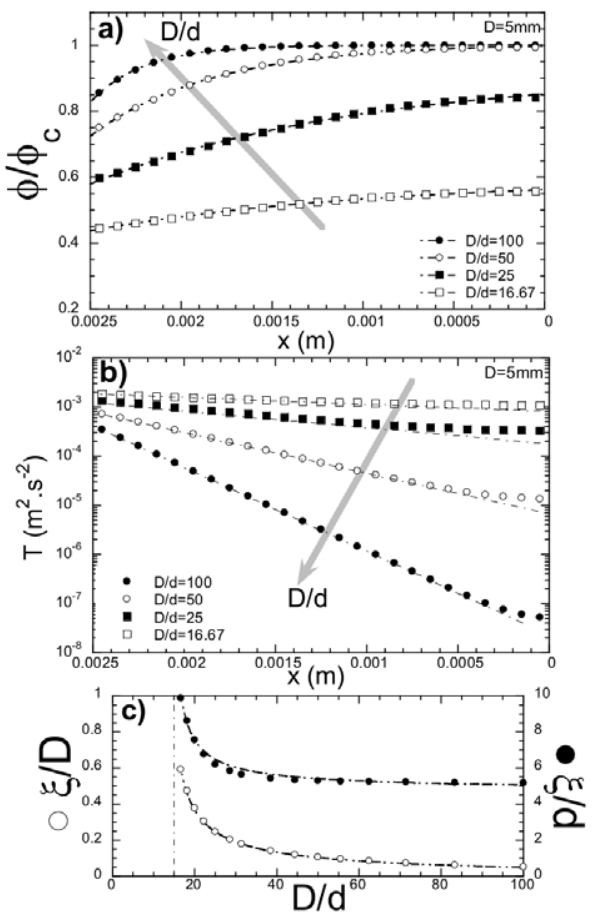

Fig. 6: Simulation results for different aspect ratios $D / d$ : (a) volume fraction profiles; (b) temperature profiles; (c) characteristic length of the exponential decrease of the temperature away from the wall (rescaled by the grain size $d$ and the domain size of the computation $D$ ) vs. the aspect ratio $D / d$.

for the high aspect ratios $D / d$. The temperature profiles displayed in fig. 6(b) show a pronounced exponential drop far from the boundaries in agreement with previously reported results in the literature [19]. The cooling is more significant for the highest aspect ratios. The characteristic length $\xi$ of the exponential decrease of the temperature (vs. the distance to the heated wall) rescaled either by the grain diameter $d$ or diameter $D$ is shown in fig. 6(c). For large aspect ratios $D / d, \xi$ is close to $5 d$ as observed in several previous investigations [19]. For values of $D / d \sim 15$, a sharp increase of $\xi$ is observed. $\xi$ may reach more than half the diameter $D$. Numerical simulations using the 2D axisymmetric equations given in [23] give similar results.

The results of these numerical simulations can be compared to the experimental data for the mean volume fraction. The results are shown in fig. 7(a) for different aspect ratios $(D / d)$ while keeping fixed $D$ or $d$. The results of the numerical simulation are superimposed to the experimental data by setting an asymptotic volume fraction $\sim 0.54$ as observed experimentally for large $D / d$. The agreement is good considering the severe simplifications used in the model ( $2 D$ vs. $3 D$ and zero mean flow). In terms of density, the Compressible-Incompressible transition occurs for $0.3<\left\langle\phi_{0}\right\rangle<0.4$. This may correspond to the transition from a low-density kinetic regime to a high-density collisional regime that occurs for $\phi \sim 0.2-0.3$ a)

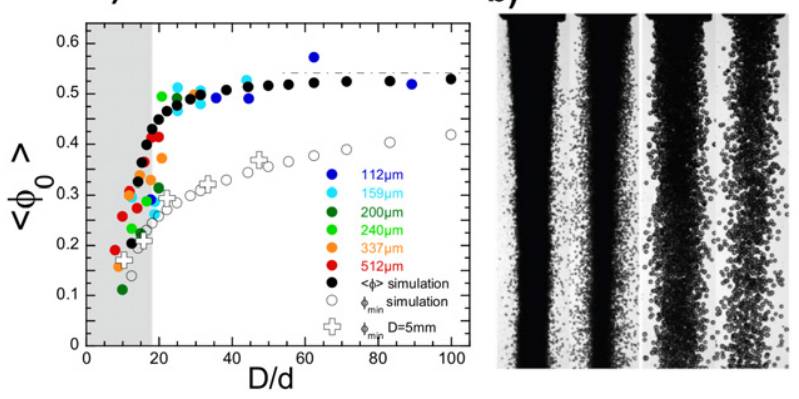

Fig. 7: (Colour on-line) (a) Mean volume fraction $\left\langle\phi_{0}\right\rangle$ at the exit of the funnel for different grain sizes $d v s$. the aspect ratio $D / d$. Black circles: results from the numerical simulation with the temperature at the wall fixed by the experimentally measured value. White crosses show the volume fraction $\phi_{\min }$ at the wall vs. $D / d$ for $D=5 \mathrm{~mm}$. White circles show $\phi_{\min }$ from the simulation. (b) For $D=13 \mathrm{~mm}$, snapshots of granular jets without and with wall roughness $(337 \mu \mathrm{m})$; first pair of images $d=337 \mu \mathrm{m}$; second pair of images $d=1000 \mu \mathrm{m}$.

as shown by Garzó and Dufty [24]. The decrease of the volume fraction observed close to $D / d \sim 15$ corroborates previous attempts to explain deviations from the empirical law of Beverloo as suggested in the literature $[7,8]$. Furthermore, as displayed in fig. 7(a), there is a good agreement with the numerical simulation if we focus on the volume fraction $\phi_{\min }$ measured experimentally near the inner wall of the funnel. To estimate $\phi_{\min }$ experimentally, we counted the number of grains $N_{\text {wall }}$ near the wall and then divided $N_{\text {wall }}$ by the volume of a truncated wedge of thickness $d$ corresponding to the measurement zone. This provides another test of the various hypotheses made for the temperature and the pressure fields in the model. We also attempted to tune the roughness at the wall boundaries in the funnel by randomly gluing grains of diameter $d \sim 337 \mu \mathrm{m}$ to the inner walls of the funnel. In this case, lower densities (than for the smooth wall funnel) are observed for a fixed aspect ratio close to the transition as displayed in fig. 7(b). This observation highlights the crucial role of the roughness at the walls: the temperature at the wall is expected to be higher for rough walls but remains difficult to measure.

Several questions remain. Our main assumption is that the temperature of the grains near the wall is fixed. But what sets the temperature near the wall? This is a difficult problem and the proper understanding of the appropriate boundary conditions remains a major challenge for continuum hydrodynamic models of granular media. One needs to solve the complete set of coupled conservation equations (mass, energy temperature and momentum [25]). Even in that case, a closure relation for the pressure has to be specified. This relation may go beyond the simple one proposed here. Our simple model also neglects any rearrangements of the grains within the bulk of the medium in the presence of flow. 
Rearrangements of the grains especially for small aspect ratios when the granular medium is flowing may also induce variations in density. In short, a full description of the transition observed here requires further theoretical work.

In conclusion, we have described a CompressibleIncompressible transition for flowing granular jets that depends solely on the aspect ratio $D / d$ at the exit of the vessel. Surprisingly, the incompressible liquid-like behavior observed in the limit of high aspect ratios seems to find its origin in the inelasticity of collisions independently of the ambient fluid pressure or the grain sizes. A simple model using continuum hydrodynamic equations to describe the granular medium seems to capture the essential features of this transition.

We would like to thank J.-F. BoudET for discussions. This research is supported by CR Aquitaine Grants No. 2006111101035, No. 20091101004 and by ANR Grant No. ANR-09-JCJC-0092.

\section{REFERENCES}

[1] Hagen G. H. L., Akad. Wiss. Berlin (1852) 35.

[2] Tighe B. P. and Sperl M., Granular Matter, 9 (2007) 141.

[3] Khamontova N., J. Russ. Phys.-Chem. Soc., 22 (1890) 281.

[4] Andreotti B., Forterre Y. and Pouliquen O., Les milieux granulaires (EDP Sciences) 2011.
[5] Beverloo W. A., Leniger H. A. and Van de Velde J. J., Chem. Eng. Sci., 15 (1961) 260.

[6] Nedderman R. M., Static and Kinematics of Granular Materials (Cambridge University Press, Cambridge) 1992.

[7] Van Zuilichem D. J., Van Egmond N. D. and De Swart J. G., Powder Technol., 10 (1974) 161.

[8] Janda A., Zuriguel I. and Maza D., Phys. Rev. Lett., 108 (2012) 248001.

[9] Eggers J. and Villermaux E., Rep. Prog Phys., 71 (2008) 036601.

[10] Rowlinson J. S. and Widom B., Molecular Theory of Capillarity (Dover) 2002.

[11] Schaflinger U. and Machu G., Chem. Eng. Technol., 22 (1999) 617.

[12] Nicolas M., Phys. Fluids., 14 (2002) 3570.

[13] Möвius M. E., Phys. Rev. E., 74 (2006) 051304.

[14] Royer J. R. et al., Nature, 459 (2009) 1110.

[15] Amarouchene Y., Boudet J. F. and Kellay H., Phys. Rev. Lett., 100 (2008) 218001.

[16] Prado G., Amarouchene Y. and Kellay H., Phys. Rev. Lett., 106 (2011) 198001.

[17] Middleman S., Modeling Axisymmetric Flows: Dynamics of Films, Jets, and Drops (Academic Press) 1995.

[18] Waitukaitis S. R. et al., Phys. Rev. E, 83 (2011) 051302.

[19] Grossman E. L., Zhou T. and Ben-Naim E., Phys. Rev. E, 55 (1997) 4200.

[20] Luding S., Nonlinearity, 22 (2009) R101.

[21] Bertho Y., Giorgiutti-Dauphiné F. and Hulin J-P., Phys. Rev. Lett., 90 (2003) 144301.

[22] Amarouchene Y. and Kellay H., Phys. Fluids., 18 (2006) 031707.

[23] Meerson B., Diez-Minguito M., Schwager T. and Pöschel T., Granular Matter, 10 (2007) 21.

[24] Garzó V. and Dufty J. W., Phys. Rev. E, 59 (1999) 5895.

[25] Khain E., EPL, 87 (2009) 14001. 